\title{
HBV cccDNA: The Stumbling Block for Treatment of HBV Infection
}

\author{
Shousheng $\operatorname{Liu}^{1}$ and Yongning Xin*2 \\ ${ }^{1}$ Central Laboratories, Qingdao Municipal Hospital, Qingdao, China; ${ }^{2}$ Department of Infectious Disease, Qingdao Municipal \\ Hospital, Qingdao, China
}

\begin{abstract}
Citation of this article: Liu S, Xin Y. HBV cccDNA: The stumbling block for treatment of HBV infection. J Clin Transl Hepatol 2019;7(3):195-196. doi: 10.14218/JCTH.2019.00047.
\end{abstract}

Hepatitis B virus (HBV) infection leads to severe liver disease and is one of the main causes of hepatocellular carcinomarelated mortality. Although the prevalence of HBV infection has decreased markedly with the effective application of vaccines, it's still an arduous task to clear the hepatitis B surface antigen after routine antiviral therapy, as the viral rebound often occurs after therapy withdrawal. Therefore, the situation of dealing with HBV infection is still challenging. ${ }^{1}$ As such, eliminating HBV infection has become an important task for governments all over the world. In 2016, the Global Health Sector Strategy on Viral Hepatitis was approved by the World Health Assembly to eliminate the HBV infection by $2030 .^{2}$

Accumulated studies have illuminated the processes underlying the HBV replication cycle, which has provided the important insight into the detailed action of the HBV lifestyle that has promoted development of therapeutic strategies against HBV infection. HBV infection begins with the lowaffinity interaction between HBV and the heparin sulfate proteoglycans located on the host hepatocytes, followed by HBV binding to the sodium taurocholate cotransporting polypeptide and then its entry into the hepatocytes. ${ }^{3}$ The HBV genome is a relaxed circular DNA with a length of $3.2 \mathrm{~kb}$, which can be transported into the nuclear compartment in its nucleocapsid form after entry into the hepatocytes.

The relaxed circular DNA can be converted into closed covalent circular DNA (cccDNA), which exists in the nuclei of infected cells and can generate offspring virus. In the nucleus, cccDNA can transcribe to HBV pregenomic RNA, S RNAs for S proteins, and X RNA for HBx protein. In the patients positive for hepatitis $B$ surface antigen (commonly referred to as HBsAb), the amounts of cccDNA could become reduced with treatment of antiviral therapy but the reduction will be modest. What's more, the cccDNA can be monitored in the liver of patients who recovered from HBV infection decades ago. ${ }^{4-6}$

\footnotetext{
Abbreviations: ccCDNA, closed covalent circular DNA; CRISPR/Cas, clustered regularly interspaced short palindromic repeats/CRISPR associated system; NAs, nucleotide analogues; HBV, hepatitis B virus; TALENs, transcription activator-like effector nucleases; ZFNs, zinc finger nucleases.

Received: 15 September 2019; Revised: 20 September 2019; Accepted: 20 September 2019

*Correspondence to: Yongning Xin, Department of Infectious Disease, Qingdao Municipal Hospital, 1 Jiaozhou Road, Qingdao, Shandong 266011, China. Tel: +86-532-82789463, Fax: +86-532-85968434, E-mail: xinyongning@163.com
}

After the HBV infection, the innate immune and adaptive immune systems are activated and initiate antiviral responses, like those involving interferon (IFN)- $\gamma$ and TNF- $\alpha$ that are produced by $T$ cells, thereby decreasing the levels of HBV ccCDNA, but the elimination of HBV cccDNA is difficult to achieve. ${ }^{7}$ As the intermediate of HBV replication, cccDNA contributes to the persistence of HBV replication due to the biological mechanism of cccDNA formation, which itself remains unclear. Eliminating the ccCDNA is critical to cure the HBV infection. ${ }^{8}$

Recently, Zhu et al. ${ }^{9}$ conducted a systemic review of HBV ccCDNA and its potential role as the therapeutic target of HBV infection. Pegylated-IFN $\alpha$ and nucleotide analogues (NAs) are the standard therapy methods, currently. Tang et al. ${ }^{10}$ reported that IFN $\alpha$ exerts a significant effect on the longterm and sustainable suppression of cccDNA transcription, which may due to the alteration of epigenetic modifications of cccDNA minichromosomes. NAs can inhibit HBV replication by targeting the viral RNA-dependent DNA polymerase, and several NAs, such as lamivudine, entecavir, telbivudine and so on, have been approved for clinical use. Although an effective antiviral response has been observed with the use of IFN $\alpha$ and NAs, the cccDNA still could not be cleared completely, at present. ${ }^{10}$

Zhu et al. ${ }^{9}$ reviewed several gene therapy strategies that have been proposed to disable HBV cccDNA (Fig. 1). The first therapeutic strategy involves the gene editing techniques, such as those based on zinc finger nucleases (ZFNs), transcription activator-like effector nucleases (TALENs), and the clustered regularly interspaced short palindromic repeats/ CRISPR associated system (CRISPR/Cas). Three ZFNs targeting the HBV polymerase, gene $X$ and core were designed and delivered into the HepAD38 cells by Weber et al., ${ }^{11}$ and they found that sustained suppression of HBV could be achieved by the HBV-targeted ZFNs in the HepAD38 cells. TALENs are similar to ZFNs and have been used in several studies to investigate their antiviral effects. The results showed that TALENs can reduce the cccDNA molecules effectively in cultured cell lines. The CRISPR/Cas system, used as a genome-editing tool, was shown to cleave DNA with sequence-specificity. Several studies have demonstrated that the CRISPR/Cas system can efficiently destroy the HBV cccDNA. Importantly, in this review, the authors pointed out some defects that should be overcome before the gene editing techniques can be applied in the clinical treatment of HBV infection. First, the specificity of the ZFNs must be ensured. Second, the TALENs should be delivered into the cells effectively. Third, the 


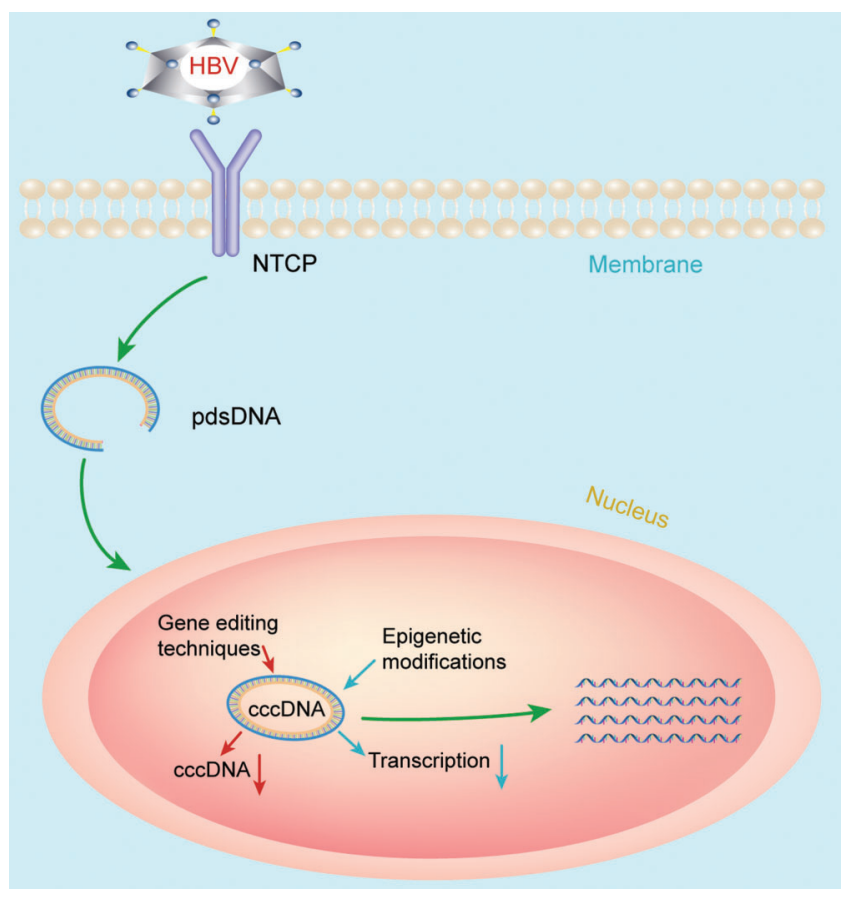

Fig. 1. Pathway of HBV infection and possible therapeutic methods of HBV infection by targeting cccDNA.

Abbreviations: cccDNA, closed covalent circular DNA; HBV, hepatitis $B$ virus.

CRISPR/Cas9 system must be delivered into the cells effectively and the off-target effect must be solved.

The second therapeutic strategy is to silence cccDNA transcription via epigenetic modifications. Results from a previous study indicated that DNA methylation and histone acetylation are required for CCCDNA, so it's promising to regulate the DNA methylation or histone acetylation to eliminate the cccDNA. Belloni et al. ${ }^{12}$ reported that the acetylation of cccDNA-bound histones could be decreased and the transcription of HBV cccDNA could be repressed by IFN $\alpha$. It has also been reported that CpG methylation can regulate the transcription of HBV cccDNA during chronic $\mathrm{HBV}$ infection, and that CpG island II methylation can decrease cccDNA transcription and subsequent viral core DNA replication. ${ }^{13}$ The acetylation status of HBV ccCDNAbound histone 3 and histone can affect the HBV replication. Wei et al. ${ }^{14}$ reported that curcumin could repress HBV replication by decreasing the cccDNA-bound histone acetylation. These conclusions suggested that epigenetic modifications could be regarded as the potential therapeutic target to eliminate HBV cCCDNA.

In summary, HBV infection, representing the most severe chronic liver disease in the world, cannot be ignored. Although some antiviral medicines, like pegylated-IFN $\alpha$ and NAs, have been approved for the clinical treatment of $H B V$, the persistent existence of cccDNA and the frequent rebound of HBV after treatment withdrawal still need to be overcome in the future. Gene editing and epigenetic modification techniques have displayed their bright prospect, but the deficiency of these therapeutic methods should be resolved in further studies. In addition, new therapeutic strategies targeting the HBV cccDNA should be explored in order to realize the goal of eliminating HBV infection by 2030 .

\section{Conflict of interest}

The authors have no conflict of interests related to this publication.

\section{Author contributions}

Article conception and design (YX), acquisition of data (SL and $\mathrm{YX})$, drafting of the manuscript (SL), critical revision of the manuscript $(Y X)$.

\section{References}

[1] Nassal M. HBV cccDNA: viral persistence reservoir and key obstacle for a cure of chronic hepatitis B. Gut 2015;64:1972-1984. doi: 10.1136/gutjnl-2015309809.

[2] Global prevalence, treatment, and prevention of hepatitis B virus infection in 2016: a modelling study. Lancet Gastroenterol Hepatol 2018;3:383-403. doi: 10.1016/S2468-1253(18)30056-6.

[3] Liang TJ, Block TM, McMahon B], Ghany MG, Urban S, Guo JT, et al. Present and future therapies of hepatitis B: From discovery to cure. Hepatology 2015;62:1893-1908. doi: 10.1002/hep.28025.

[4] Chan HL, Wong VW, Tse AM, Tse $\mathrm{CH}$, Chim AM, Chan HY, et al. Serum hepatitis $B$ surface antigen quantitation can reflect hepatitis $B$ virus in the liver and predict treatment response. Clin Gastroenterol Hepatol 2007; 5:1462-1468. doi: 10.1016/j.cgh.2007.09.005.

[5] Gao Y, Li Y, Meng $Q$, Zhang Z, Zhao $P$, Shang $Q$, et al. Serum hepatitis B virus DNA, RNA, and HBsAg: Which correlated better with intrahepatic covalently closed circular DNA before and after nucleos(t)ide analogue treatment? J Clin Microbiol 2017;55:2972-2982. doi: 10.1128/JCM.00760-17.

[6] Testoni B, Durantel D, Zoulim F. Novel targets for hepatitis B virus therapy. Liver Int 2017;37 Suppl 1:33-39. doi: 10.1111/liv.13307.

[7] Xia Y, Stadler D, Lucifora J, Reisinger F, Webb D, Hösel M, et al. Interferon- $\gamma$ and tumor necrosis factor- $\alpha$ produced by T cells reduce the HBV persistence form, cccDNA, without cytolysis. Gastroenterology 2016;150:194-205. doi: 10.1053/j.gastro.2015.09.026.

[8] Ni Y, Lempp FA, Mehrle S, Nkongolo S, Kaufman C, Fälth M, et al. Hepatitis B and $D$ viruses exploit sodium taurocholate co-transporting polypeptide for species-specific entry into hepatocytes. Gastroenterology 2014;146:10701083. doi: $10.1053 /$ j.gastro.2013.12.024.

[9] Zhu A, Liao X, Li S, Zhao H, Chen L, Xu M, et al. HBV cccDNA and its potential as a therapeutic target. J Clin Transl Hepatol 2019;7:258-262. doi: 10. 14218/JCTH.2018.00054.

[10] Tang LSY, Covert E, Wilson E, Kottilil S. Chronic hepatitis B infection: A review. JAMA 2018;319:1802-1813. doi: 10.1001/jama.2018.3795.

[11] Weber ND, Stone D, Sedlak RH, De Silva Feelixge HS, Roychoudhury $P$, Schiffer JT, et al. AAV-mediated delivery of zinc finger nucleases targeting hepatitis B virus inhibits active replication. PLoS One 2014;9:e97579. doi: 10.1371/journal.pone.0097579.

[12] Belloni $L$, Allweiss $L$, Guerrieri $F$, Pediconi N, Volz T, Pollicino T, et al. IFN- $\alpha$ inhibits HBV transcription and replication in cell culture and in humanized mice by targeting the epigenetic regulation of the nuclear cccDNA minichromosome. J Clin Invest 2012;122:529-537. doi: 10.1172/JCI58847.

[13] Zhang Y, Mao R, Yan R, Cai D, Zhang Y, Zhu H, et al. Transcription of hepatitis $B$ virus covalently closed circular DNA is regulated by $C p G$ methylation during chronic infection. PLoS One 2014;9:e110442. doi: 10.1371/journal.pone. 0110442.

[14] Wei ZQ, Zhang $\mathrm{YH}, \mathrm{Ke} C Z$, Chen $\mathrm{HX}$, Ren $\mathrm{P}$, He $\mathrm{YL}$, et al. Curcumin inhibits hepatitis $B$ virus infection by down-regulating cccDNA-bound histone acetylation. World J Gastroenterol 2017;23:6252-6260. doi: 10.3748/wjg.v23. i34.6252. 